\title{
Antenna Gain Measurements by an Extended Version of the NBS Extrapolation Method
}

\author{
ANDREW G. REPJAR, SENIOR MEMBER, IEEE, ALLEN C. NEWELL, MEMBER, IEEE, \\ AND RAMON C. BAIRD, SENIOR MEMBER, IEEE
}

\begin{abstract}
A General Extrapolation Technique which corrects for the effects of ground reflections in absolute gain measurements is described. It utilizes the Extrapolation Method developed at NBS which, in its present form, utilizes only amplitude versus distance data. However, for broadbeam antennas such as those encountered below $1 \mathrm{GHz}$, ground reflections may produce unwanted oscillations in the amplitude versus distance data. Hence the data are not amenable to the curve-fitting procedure of the Extrapolation Method. This problem can be overcome by including phase versus distance information to reduce the effects of ground reflections.
\end{abstract}

\section{INTRODUCTION}

A SPECIFIC OBJECTIVE of the Antenna Systems Metrology Group at NBS is to develop the required techniques and standards for accurately determining antenna characteristics. One existing NBS measurement service is the accurate calibration of transfer standard antennas (on-axis gain and polarization) by the Extrapolation Method. The theoretical basis of this method was developed by Wacker [1] and its application to accurate antenna measurements has been described briefly by Newell and Kerns [2] and more fully, in an experimentally oriented paper, by Newell et al. [3]. The method utilizes a generalized three-antenna approach which does not require quantitative $a$ priori knowledge of the antennas.

The main advantages of the Extrapolation Method, which presently utilizes amplitude versus distance data only, are its accuracy and generality, and there is essentially no upper frequency limit. It can, in principle, be applied to any type of antenna although some directivity is desirable to reduce multipath interference. Above $1 \mathrm{GHz}$, uncertainties between \pm 0.08 and $\pm 0.10 \mathrm{~dB}$ in gain are achieved routinely. The maximum gain values that can be measured depend on the range dimensions since a maximum separation of approximately $2 D^{2} / \lambda$ is desirable. With the present NBS facilities, the maximum gain ranges from about $45 \mathrm{~dB}$ at $75 \mathrm{GHz}$ down to about $25 \mathrm{~dB}$ at $1 \mathrm{GHz}$.

At frequencies below $1 \mathrm{GHz}$ and for the purposes of this paper, in the VHF-UHF region, a transfer standard is often broadbeam and produces ground reflections which cause oscillations in the amplitude data as a function of distance. These oscillations have longer periods than those due to the multiple reflections between antennas which are routinely averaged out

Manuscript received August 19, 1982.

The authors are with the Antenna Systems Metrology Section, Electromagnetic Fields Division, National Bureau of Standards, Boulder, CO 80303.

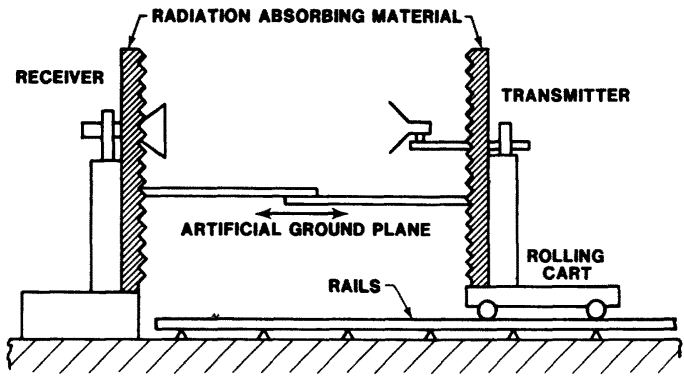

Fig. 1. Schematic diagram showing the NBS indoor extrapolation range modified to function as a scaled model of the larger outdoor range.

in the Extrapolation Method. For this reason, below $1 \mathrm{GHz}$ and for gains less than about $15 \mathrm{~dB}$, the antenna might not be calibratable by the usual Extrapolation Method which uses a curve-fitting procedure to determine gain. The purpose of this paper is to demonstrate that by utilizing both amplitude and phase data as a function of distance, one can obtain an amplitude versus distance curve which is corrected for the effects of ground reflections. This curve can then be processed by the Extrapolation Method to determine gain.

\section{Discussion of Measurement Procedure}

Recently at NBS, it was determined that a VHF-UHF scalar horn can be designed to meet the requirements of a transfer standard for $240-400 \mathrm{MHz}$ [4]. This fact was demonstrated by designing and constructing a $C$-band model of the VHF-UHF antenna and extensively measuring the critical performance parameters and comparing them with theoretical results. The expected calibration accuracy of the VHF-UHF horn was then determined by performing measurements of the scaled model on the NBS indoor extrapolation range. Measurements were conducted both with and without ground reflections so that we could determine how well we could correct for the multipath effects in our calibration technique. Ultimately, the full-scale horn will be measured on the NBS outdoor extrapolation range. Fig. 1 is an illustration of how we can use the scaled model horn on the indoor range to simulate the situation that will exist at $240-400 \mathrm{MHz}$ on the outdoor range.

\section{EXTRAPOLATION METHOD}

The first step in the measurement process is to connect the generator and load ports together to obtain a reference signal. The antennas are then installed and the received signal is re- 


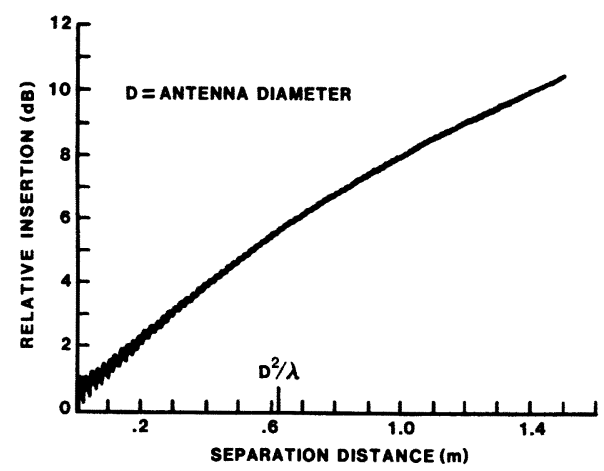

Fig. 2. Extrapolation data for scalar horn showing the insertion loss plotted as a function of separation distance (meters).

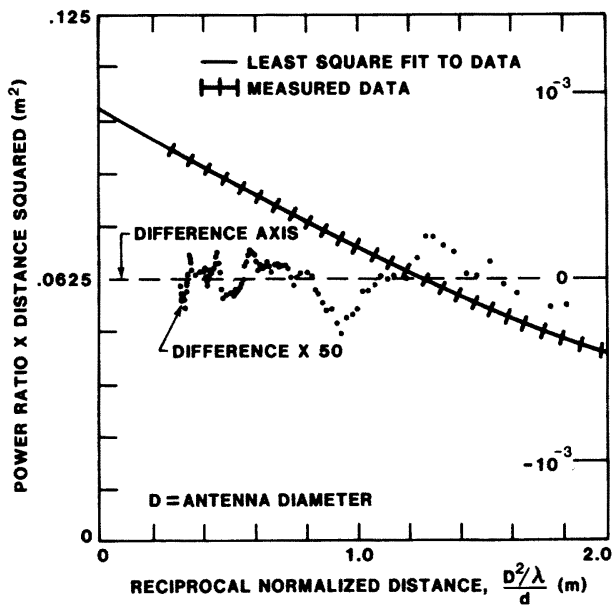

Fig. 3. Measured data without ground plane, four-term polynomial fit, and differences for scalar horn.

corded as a function of the separation distance. Fig. 2 displays some typical data for the case where no ground reflections are present. The oscillations are due to multiple reflections between the antennas. In the curve-fitting process to determine gain, the effects of these multiple transits are eliminated by locally averaging the relative insertion-loss values. The averaged curve is then fitted with a polynomial in $1 / d$ (ordinarily, four or five terms will suffice). This procedure determines the coefficient, $A_{00}$, of the 1/d term of the series (see [3], eq (27)] which determines the gain of the antenna under test. With the artificial ground plane removed and, hence, with no ground reflections present, the results obtained by the curve-fitting procedure are given in Fig. 3, which shows the averaged curve obtained from the measured data for the scalar horn and the curve obtained by fitting with a four-term polynomial. The greatly magnified differences between the measured and fitted curve qualitatively indicate the goodness of fit. The absolute gain of this horn was determined to be $13.32 \mathrm{~dB}$ at $4.0 \mathrm{GHz}$, which simulates 240 $\mathrm{MHz}$ in the VHF-UHF range. It should be noted that the abscissa in Fig. 3, and subsequently in Figs. 4 and 6, is the inverse of the normalized separation distance, a natural means of analyzing curve fitting with a polynomial in $1 / d$.

\section{ANALYSIS}

With the artificial ground plane in place, the averaged curve, i.e., the curve obtained after applying the local averaging process discussed above, as a function of antenna separation

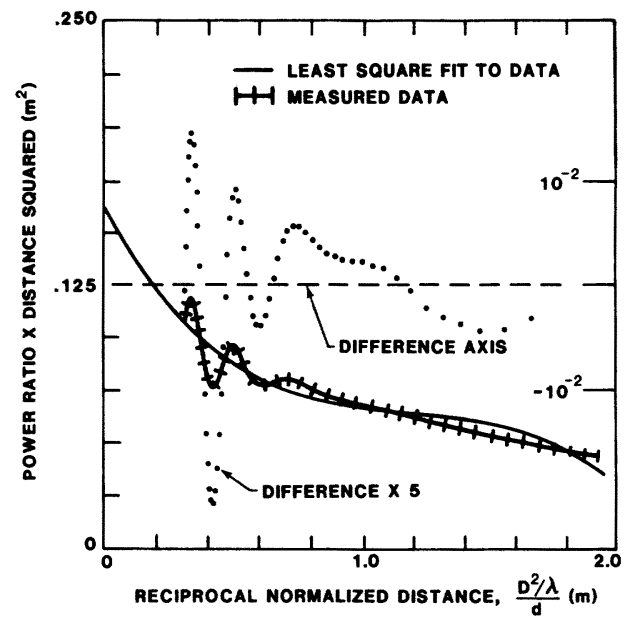

Fig. 4. Measured data with ground plane, four-term polynomial fit, and differences for scalar horn.

is given in Fig. 4. Note the region of oscillatory behavior due to ground reflections. Fitting this curve with a four-term polynomial in $1 / d$, which is also shown in Fig. 4 , results in a calculated gain of $14.29 \mathrm{~dB}, 0.97 \mathrm{~dB}$ higher than its calibrated value obtained without the ground plane! In addition, it is evident from this figure that the differences between the averaged curve and the fitted curve are pronounced.

Denoting $\phi_{t}$ as the measured phase and $\phi_{d}$ as the phase of the direct wave, one can see that when the ground plane is removed, i.e., no ground effects present, the measured phase minus the phase of the direct wave should be zero plus an arbitrary constant in the far field. This is important in our analysis because the only assumption made in obtaining an amplitude versus distance plot that is free of multipath effects is that the direct wave phase can be represented in the far field as

$$
\phi_{d}=360 d / \lambda \text { degrees }
$$

where $d$ is the separation distance and $\lambda$ is the wavelength.

Using a laser interferometer to accurately measure the separation distance, the phase was measured without the ground plane to test the above assumption. (Since (1) is being used to calculate the phase of the direct wave, the measured separation distance must be accurate to about $1 / 360$ of a wavelength. For this reason, at $C$-band, a laser interferometer was used to measure the separation distances. For measurements of the full-scale UHF-VHF horn, other means may be adequate.) The dashed line in Fig. 5 shows the results, demonstrating that for large distances, $\phi_{t}-\phi_{d}$ is in fact extremely close to being constant. It should be noted that where the separation distance is small, the variation of $\phi_{t}-\phi_{d}$ is due to the near-zone effect, i.e., the antennas are still in the near field of each other. (A correction for this effect is routinely obtained in the standard extrapolation method.)

An analysis of the interaction between the direct wave and the ground wave shows that at the local peaks of the $\phi_{t}-\phi_{d}$ curve

$$
\rho \equiv \frac{\left|V_{g}\right|}{\left|V_{d}\right|}=\sin \left(\phi_{t}-\phi_{d}\right)
$$

where $\left|V_{g}\right|$ and $\left|V_{d}\right|$ are the amplitudes of the ground and direct waves, respectively. In addition, it can be shown that 


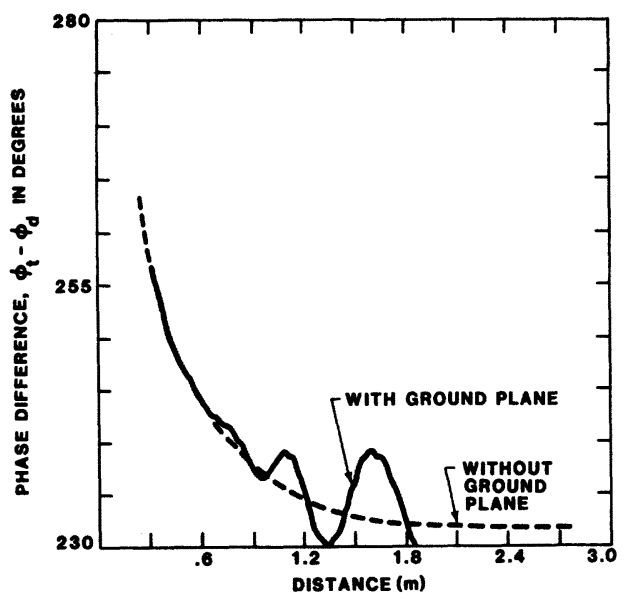

Fig. 5. Phase difference between measured wave and direct wave with and without ground plane.

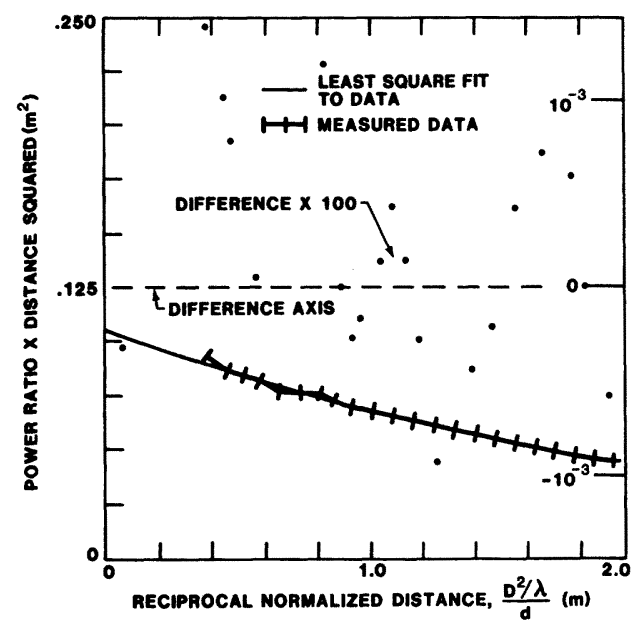

Fig. 6. Measured data points with ground plane and $\left|V_{t}\right|=\left|V_{d}\right|$, fourterm polynomial fit, and differences for scalar horn.

$$
\frac{\phi_{t}-\phi_{d}}{2}=\sin ^{-1}(\rho / 2), \quad \text { when }\left|V_{t}\right|=\left|V_{d}\right|
$$

where $\left|V_{t}\right|$ is the amplitude of the total wave resulting from the combination of the direct and ground waves. It is $\left|V_{t}\right|$ that is measured as a function of distance in the extrapolation method. Since $\left|V_{t}\right|_{\max }$ and $\left|V_{t}\right|_{\min }$ occur when the ground wave adds in and out of phase, respectively, with the direct wave, the distances at which $\phi_{t}-\phi_{d}=0$ are also determined. From these preceding results, the points on the insertion-loss curve where $\left|V_{t}\right|=\left|V_{d}\right|$ can be determined. For example, when the phase differences, $\phi_{t}-\phi_{d}$, are small, the condition $\left|V_{t}\right|=\left|V_{d}\right|$ occurs at those distances where the $\phi_{t}-\phi_{d}$ curve has local maxima and minima. Using only these points as data in the region where ground reflection effects are dominant, one can then use the extrapolation method to determine gain.

The solid line in Fig. 5 displays the measured $\phi_{t}-\phi_{d}$ data obtained with the ground plane in place. Through the above procedure, the points where $\left|V_{t}\right|=\left|V_{d}\right|$ were determined, and the curve obtained using these points in the region where ground effects are dominant is shown in Fig. 6. After fitting this curve with a four-term polynomial, also shown in Fig. 6, the gain of the antenna was calculated to be $13.27 \mathrm{~dB}$, only $0.05 \mathrm{~dB}$ lower than the value obtained in the absence of ground reflections! In addition, in this figure, the differences between the curve and the fitted curve indicate the goodness of fit.
TABLE I

MEASUREd Gain IN dB OF THE SCAlar Horn MOdel (Principal component horizontally oriented.)

\begin{tabular}{cccc}
\hline \hline $\begin{array}{c}\text { Frequency } \\
\text { in GHz }\end{array}$ & Without ground plane & With ground plane & $\begin{array}{c}\text { With ground plane } \\
\text { and back-structure }\end{array}$ \\
\hline 4.0 & 13.32 & 13.27 & 13.25 \\
4.83 & 14.10 & 14.11 & 14.15 \\
5.5 & 14.52 & 14.41 & 14.41 \\
6.8 & 12.15 & 12.17 & 12.22 \\
\hline
\end{tabular}

TABLE II

MEASURed Gain in dB OF SCALAR HORn MODEl (Principal component vertically oriented.)

\begin{tabular}{cccc}
\hline \hline $\begin{array}{c}\text { Frequency } \\
\text { in GHz }\end{array}$ & Without ground plane & With ground plane & $\begin{array}{c}\text { With ground plane } \\
\text { and back-structure }\end{array}$ \\
\hline 4.0 & 13.23 & 13.31 & 13.31 \\
4.83 & 14.09 & 14.02 & 14.03 \\
5.5 & 14.41 & 14.50 & 14.54 \\
6.8 & 12.17 & 12.26 & 12.29 \\
\hline
\end{tabular}

\section{RESULTS}

In order to further test the use of the extended version of the extrapolation method, the gain of the scaled-model antenna was determined at four frequencies across the band. The amplitude versus distance data obtained without the ground plane present were processed by the extrapolation method to determine the gain of the antenna at these frequencies. Next, the anplitude versus distance data obtained with the ground plane present were processed by the extended version of the extrapolation method to determine the gain. Comparisons were then made. In addition, the gain of the antenna was obtained with its principal component first horizontally and then vertically oriented. (The phase of the field reflected by the ground plane depends on the polarization of the field incident on the ground plane. This results in different, i.e., shifted, positions for the oscillations in the amplitude versus distance data. To be useful, the extended version of the extrapolation method must give the correct gain for all orientations.) Finally, the structures of the towers on which antennas are mounted on the outdoor range were simulated to determine their effect on the gain measurements. The results of all these measurements are summarized in Tables I and II.

Upon examination of the data in Tables I and II, the rms difference between the gain values obtained with and without the ground plane are 0.07 and $0.09 \mathrm{~dB}$ for the principal component horizontally and vertically oriented, respectively. Also, the rms difference between the gain values obtained without the ground plane for the horizontal and vertical orientations is $0.07 \mathrm{~dB}$. These values are within $\pm 0.10 \mathrm{~dB}$, the bounds nominally expected by the error analysis discussed in [3]. It should be again noted that without the extended version of the extrapolation method to account for ground reflections, errors in gain could be in the order of \pm 1 to $2 \mathrm{~dB}$.

\section{CONCLUSIONS}

A technique has been described to determine the gain of antennas operating at frequencies below $1 \mathrm{GHz}$. Under simulated conditions, it was determined that phase versus distance measurements can be used to determine an amplitude versus distance curve which is corrected for ground reflection effects. This curve can then be processed by the extrapolation method which uses amplitude data only to determine gain. Measure- 
ments on the full-scale VHF-UHF horn on the NBS outdoor extrapolation range are planned for the future.

\section{REFERENCES}

[1] P. F. Wacker, "Theory and numerical techniques for accurate extrapolation of near-zone antenna and scattering measurements," NBS, Boulder, CO, unpublished Rep., Apr. 1972.

[2] A. C. Newell and D. M. Kerns, "Determination of both polarization and power gain of antennas by a generalized 3-antenna measurement method," Electron Lett., vol. 7, pp. 68-70, Feb. 11, 1971.

[3] A. C. Newell, R. C. Baird, and P. F. Wacker, "Accurate measurement of antenna gain and polarization at reduced distances by an extrapolation technique," IEEE Trans. Antennas Propagat., vol. AP-21, no. 4, pp. 418-431, July 1973.

[4] A. J. Estin, C. F. Stubenrauch, A. G. Repjar, and A. C. Newell, "Optimized wavelength-sized scalar horns as antenna radiation standards," IEEE Trans. Instrum. Meas., vol. IM-31, no. 1, pp. 53-56, Mar. 1982.

\title{
A Near-Field Antenna Measurement System
}

\author{
JOHN BOROWICK, MEMBER, IEEE, ALAN E. HOLLEY, MEMBER, IEEE, W. L. LANGE, \\ R. W. HOWARD, AND R. L. CUMMINGS
}

\begin{abstract}
This paper describes the development, operation, and performance of a fully automated self-contained system which measures the near-field phase and amplitude of an antenna aperture and then computes and displays far-field antenna patterns and related data.
\end{abstract}

\section{PURPOSE AND CONCEPT}

$\mathrm{T}$ HE MEASUREMENT of antenna characteristics is a significant cost item in the development, manufacturing, and field use of many radar and communications systems. These costs are particularly high in the case of complex phased-array systems where large amounts of data are required for adequate evaluation. Conventional far-field antenna measurements, even when automated, require a lengthy program if a complete evaluation is to be properly carried out. If problems arise, the data that can be obtained on a far-field range rarely give adequate insight into the difficulty. A single miswired phase shifter may be almost impossible to find without dismantling the system.

The near-field measurement concept, which was developed into a practical approach by the National Bureau of Standards and the Georgia Institute of Technology provides an effective alternative to the far-field range. A near-field system has been developed which is completely self-contained and, after initialization, measures and evaluates the performance of pro-

Manuscript received August 19, 1982. This work was performed under USA ERADCOM Contract DAAB07-77-C-0587 with the Combat Surveillance and Target Acquisition Laboratory, Ft. Monmouth, NJ. It was accomplished as part of the U.S. Army Manufacturing and Technology Program.

J. Borowick is with the CS\&TA Laboratory USA ERADCOM, Ft. Monmouth, NJ 07703.

A. E. Holley, W. L. Lange, R. W. Howard, and R. L. Cummings are with Hughes Aircraft Company, Fullerton, CA 92634. duction phased-array systems without operator interaction. While the primary use is production testing, the system program capabilities include general-purpose antenna evaluation and automated diagnostic testing. The near-field cost advantage arises from two factors; first the data obtained during a single two-dimensional scan of the aperture give, when analyzed, the entire two-dimensional pattern as well as beam position, gain, and other antenna parameters. In addition, the ability to measure many multiplexed data sets during that single scan means that an entire performance test may be completed in one measurement cycle. In practical terms, a group of 150 combinations of frequency, beam position, and port can be measured in about $14 \mathrm{~h}$ with an analysis for production evaluation requiring only another $4 \mathrm{~h}$. Thus a complete cycle of testing can be completed in one day.

\section{SYSTEM DESCRIPTION}

The near-field system consists of a two axis probe positioner, a mounting structure for the antennas to be tested, a signal source, a phase-amplitude detector, a computer system which includes all of the display and control equipment, and the special software for measurement, analysis, and display.

The probe positioner is similar to that used by NBS and may be thought of as a large $X Y$ plotter standing on end. Fig. 1 shows the positioner shortly after initial assembly. The probe can travel over a range of $13 \mathrm{ft}$ in the $X$ (horizontal) direction and $15 \mathrm{ft}$ in the $Y$ (vertical) direction. A corner of the antenna mounting structure can also be seen in this figure. It consists of a heavy-duty rotatable base which moves at right angles to the $X Y$ plane on roundway bearings. This combination of movement in the $Z$ direction and rotation about the $Y$ axis 